\title{
Hábitos de Higiene Oral en Estudiantes de Odontología de la Universidad de Chile
}

\author{
Oral Health Habits in Dental Students at the University of Chile
}

\author{
Espinoza Santander I1 , Muñoz Poblete $\mathrm{C}^{2}$, Lara Molina $\mathrm{M}^{3}$, Uribe Cifuentes $\mathrm{JP}^{4}$
}

\begin{abstract}
RESUMEN
Los estudiantes de odontología son agentes involucrados en la educación de los cuidados bucodentales. No se han publicado estudios nacionales que describan sus hábitos de higiene oral. Objetivo: Describir la proporción de estudiantes de odontología de la Universidad de Chile con hábitos de higiene oral saludables según sexo, nivel socioeconómico y años de estudios. Material y método: Estudio transversal descriptivo. Se seleccionaron, por muestreo aleatario simple con afijación proporcional 150 estudiantes entre 1er- 4to año. Se aplicó un cuestionario sobre hábitos de higiene oral, que incluía uso y frecuencia de cepillado, seda dental y enjuagatorio bucal, tiempo transcurrido desde la última visita al dentista y variables sociodemográficas. Se construyó un índice Hábitos de Higiene Oral que incluía cepillado por lo menos dos veces al día, uso de seda dental diariamente y visita al dentista por lo menos una vez por año. Resultados: Un $98 \%$ se cepillaba los dientes por lo menos dos veces al día, $37 \%$ usaba seda dental diariamente y $74 \%$ había acudido al dentista por lo menos una vez en el último año. Sólo un 30\% presentó un resultado positivo para el índice Hábitos de Higiene Oral. No se detectaron diferencias significativas según sexo, nivel socioeconómico y años de estudios. Conclusiones: Los estudiantes de odontología de la Universidad de Chile presentan alta frecuencia de cepillado dental y consultan frecuentemente al dentista, sin embargo, el uso de seda dental es bajo. Estudios futuros deberían analizar otras variables involucradas en un mejor cuidado de la salud bucal en este grupo.
\end{abstract}

Rev. Clin. Periodoncia Implantol. Rehábil. Oral Vol. 3(1); 11-18, 2010.

Palabras clave: Salud oral, estudiantes de odontología.

\section{ABSTRACT}

Dental students are agents involved in the education of oral care. There are no published studies that describe their oral hygiene habits in Chile. Objective: To describe the proportion of dental students at the University of Chile with healthy oral hygiene habits by sex, socioeconomic status and years of study. Material and methods: A descriptive cross-sectional study on 150 students from 1st-4th year of dentistry studies were selected by simple sampling with proportional allocation. A questionnaire on oral hygiene habits that included questions about the use and frequency of brushing and flossing, mouthwashes use, time since the last dental visit and sociodemographic variables were used. An index about oral hygiene was constructed including brushing at least twice a day, flossing at least once a day and visiting your dentist at least once a year. Results: About $98 \%$ of students brushing teeth at least twice daily, $37 \%$ used dental floss daily and $74 \%$ had visited the dentist at least once in the past year. Only $30 \%$ of students had a positive outcome for the oral hygiene index. No significant differences by gender, socioeconomic status and years of study were observed. Conclusions: Dental students at the University of Chile show high frequency of tooth brushing and frequently visit to the dentist, however, flossing is low. Future studies should also analyze other variables involved in better oral health care in this group.

Rev. Clin. Periodoncia Implantol. Rehábil. Oral Vol. 3(1); 11-18, 2010.

Key words: Oral health, dental students.

\section{INTRODUCCIÓN}

Las patologías bucales son frecuentes en la población en Chile. Según la Encuesta Nacional de Salud el $66 \%$ las personas adultas tiene caries dentales y un $33 \%$ de los mayores de 65 años han perdido todos sus dientes principalmente debido a caries ${ }^{(1)}$, lo que junto a la estimación de que cerca de un $100 \%$ de la población presenta enfermedad periodontal (2), nos da cuenta de la gran magnitud de este problema. Diversos factores se asocian con una mayor prevalencia de las enfermedades bucodentales como pobreza, limitada disponibilidad y accesibilidad a los servicios de salud y de flúor en el agua y, variables relacionadas a los estilos de vida no saludables como una nutrición inadecuada, uso de tabaco y alcohol y, principalmente, una deficiencia en los hábitos de higiene oral, como cepillado dental(3).

Dentro de los factores que influyen en la adquisición de hábitos de higiene oral adecuados se encuentran las creencias personales, valores, actitudes, autoeficacia, influencia de los miembros de la familia, amigos, compañeros de trabajo y líderes de opinión ${ }^{(4)}$ y un aspecto escasamente explorado que es el rol del personal de salud, sobre el cual se ha descrito que sus creencias y actitudes afectan su habilidad para lograr persuadir a sus pacientes para que cuiden adecuadamente su salud bucal ${ }^{(5,6)}$.
En nuestro país, no encontramos publicaciones indexadas sobre hábitos de higiene oral en odontólogos o en estudiantes de odontología. Sin embargo, en la población general de adolescentes entre 15 y 20 años se ha descrito que aproximadamente un $30 \%$ tiene hábitos de higiene oral deficientes, por ejemplo, se cepilla sus dientes menos de dos veces al día ${ }^{(7)}$. Debido al rol que cumplen los estudiantes de odontología, durante el transcurso de su formación y luego en su ejercicio profesional, en la promoción, prevención y tratamiento de las enfermedades bucodentales, es interesante analizar cuáles son sus hábitos de higiene oral.

El objetivo del presente estudio es describir la proporción de estudiantes de odontología de la Universidad de Chile matriculados el año 2009 con hábitos de higiene oral saludables y analizar cómo se comporta esta proporción según sexo, nivel socioeconómico y años de años de formación profesional de pregrado.

\section{MATERIAL Y MÉTODO}

Este estudio corresponde a uno de tipo transversal descriptivo para el cual se tomó en cuenta la población de los estudiantes

\footnotetext{
1. Magíster en Patología Oral, Alumna del Programa de Doctorado en Salud Pública, Universidad de Chile. Académico de la Facultad de Odontología. Universidad de Chile. Chile.

2. Magíster en Salud Ocupacional, Alumno del Programa de Doctorado en Salud Pública, Universidad de Chile. Académico de la Facultad de Medicina, Universidad de la Frontera. Chile.

3. Nutricionista, Alumna del Programa de Magíster en Nutrición. Universidad de Chile. Chile.

4. Geógrafo, Alumno del Programa de Magíster en Salud Pública. Universidad de Chile. Chile.

Correspondencia autor: Iris Espinoza S. Escuela de Salud Pública. Universidad de Chile. Independencia No 639. Independencia, Chile. Fono: (56-2) 9786540. iespinoz@uchile.cl / irisespinoza.patologiaoral@gmail.com. Trabajo recibido el 23/03/2010. Aprobado para su publicación el 17/04/2010.
} 
universitarios matriculados en la carrera de odontología en el año 2009 , 605 alumnos hasta la fecha del 7de Abril del año 2009.

La técnica de muestreo consideró un procedimiento aleatorio estratificado. De acuerdo a la estratificación por sexo y por año de formación profesional se seleccionaron los sujetos según curso y en cada uno de ellos se ejecutó una selección aleatoria simple. La asignación de los elementos a cada uno de los estratos que componen la muestra se realizó bajo el criterio de afijación proporcional, de manera que el tamaño de cada estrato de la muestra sea proporcional al tamaño del correspondiente estrato de la población en estudio.

El cálculo del tamaño muestral se realizó considerando un universo de 605 alumnos matriculados en el año 2009 y una proporción esperada de hábitos de higiene oral de un $30 \%(7)$, con un $95 \%$ de confianza y un $5 \%$ de precisión. La muestra final quedó constituida por 150 alumnos de 1 ero a 4to año de odontología.

A través de la Encuesta (Anexo 1) se estudiaron variables explicativas tales como edad, sexo, escolaridad paterna y materna, promedio de los ingresos del grupo familiar, años de estudio, año de carrera que actualmente cursa y como variable respuesta se considero a los hábitos de higiene oral saludables definido conceptualmente como las prácticas realizadas para mantener la limpieza de los dientes, encías y mucosa oral y evitar el desarrollo y complicaciones de enfermedades orales de tipo infecciosas, principalmente caries y enfermedad periodontal. Para la operacionalización de esta variable se consideró que un estudiante tiene Hábitos de Higiene Oral Saludable cuando obtenía un puntaje de 3 puntos al sumar las dimensiones cepillado dental (2 veces al día o más), uso de seda dental (al menos una vez al día) y visita al dentista (al menos una vez al año).

Se construyó un índice de nivel socioeconómico a partir de las variables escolaridad de la madre y del padre, e ingreso total mensual del grupo familiar. Se construyeron tres niveles socioeconómicos: el alto considera ingresos del grupo familiar de mas de dos millones y educación técnica y universitaria competa o incompleta para la madre y el padre, el nivel socioeconómico medio considera ingresos familiares entre 500.001 pesos a 2.000.000 pesos y educación media completa e incompleta para madre y padre y el nivel bajo considero ingresos hasta 500.000 pesos y educación básica completa e incompleta de los padres.

Previo a la recolección de datos se construyeron los siguientes instrumentos: manual de procedimientos, cuestionario, hoja de codificación de datos, cuaderno de recogida de datos, instructivo de entrenamiento del personal que entregará las encuestas y carta de consentimiento informado.

Se accedió a los estudiantes de odontología de la Universidad de Chile, a través de una coordinación con el Centro de Estudiantes de Odontología (CEO) y los profesores de la carrera, solicitando un espacio de horario en la clase. A los alumnos seleccionados para el estudio se les explicó que la participación era voluntaria, pudiendo desistir de responder el cuestionario en cualquier momento, a todos se les solicitó leer un consentimiento que informaba sobre las características del estudio y se les pidió que lo firmaran si aceptaban colaborar. Cabe destacar, que no se logró encuestar a los alumnos de 5 to y 6 to año debido a dificultades para acceder a ellos.

Se capacitaron dos personas encuestadoras en el propósito del estudio, procedimiento a realizar, instrumento a aplicar, manejo y almacenamiento de las encuestas para su posterior devolución a encargado del ingreso de los datos. La aplicación del instrumento fue de manera auto-administrada, bajo la supervisión de un encuestador que podrá resolver dudas y asegurar la recepción y almacenaje de los cuestionarios al concluir el llenado.

Se planificó un estudio piloto, aplicando el cuestionario a 5 personas y se solicitó comentar de manera escrita o verbal las dificultades de comprensión de preguntas o de las categorías de las respuestas, sugerencias gramaticales, ortográficas y de orden lógico.

Para el análisis de los datos obtenidos de las encuestas fueron ingresadas en una base de datos por dos parejas de digitadores, mediante el programa de doble verificación Epidata ${ }^{\circledR}$.

La proporción de estudiantes que tiene los diferentes hábito de higiene oral (frecuencia de cepillado, uso de seda dental y visitas al dentista) y los que tienen el Índice Habito de Higiene Oral Saludable (si/no) se describieron utilizando porcentajes en el total de la muestra y según las variables edad, sexo, educación de los padres y años de estudio. Para cada una de las categorías de higiene oral se utilizarán porcentajes. Para la variable descriptiva edad se utilizó como medida de tendencia central la mediana y para las otras variables que describen la muestra se utilizaron porcentajes.

Se utilizó el Software STATA 8.0 para el procesamiento y análisis de los datos obtenidos y se realizarán inferencias estadísticas con un $95 \%$ de confianza.

\section{RESULTADOS}

La variable edad en la muestra no presentó una distribución normal. Las edades de los participantes se encontraban entre los 18 y 31 años con una mediana de 20 años y un rango intercuartilico $(25-75 \%)$ de 19-21 años. El mayor porcentaje de alumnos tiene la edad de 19 años $(24 \%)$.

La distribución por sexo en los estudiantes encuestados fue similar. Debido a que el método de muestreo utilizado en el estudio fue de afijación proporcional, la muestra quedo conformada por una mayor proporción de estudiantes de primer año en comparación al resto debido a que hay un mayor número de alumnos matriculados en 1er año, y por la misma razón se obtuvo una menor proporción de estudiantes de cuarto año. Como se puede observar en la Tabla 1 el mayor porcentaje de los alumnos se clasificó como de nivel socioeconómico alto $(50,6 \%)$ y el menor porcentaje en nivel socioeconómico bajo $(18,3 \%)$.

En relación a las variables descriptoras de comportamiento en el cuidado de la salud oral, casi la totalidad de los estudiantes presentaron una la frecuencia de cepillado dental saludable (dos veces o más al día). Respecto al uso de seda dental este hábito se presentó en un mayor porcentaje como un comportamiento no saludable $(62,7 \%)$, dado que un porcentaje minoritario la utilizó diariamente. Respecto a la variable última visita al dentista hace menos de 1 año se detectó que un alto porcentaje $74 \%$ lo había realizado.

La descripción de todas las variables consideradas en el estudio, se presenta en los Anexos 2 y 3

Considerando estas 3 variables anteriormente mencionadas se construyó un índice de hábitos de higiene oral saludables, los resultados mostraron que sólo un $30 \%$ de los estudiantes de Odontología de la Universidad de Chile presentaron un valor de índice positivo o saludable para estas 3 variables (Tabla 2).

En la Tabla 3 se observa la distribución del Índice Hábitos de Higiene Oral Saludables según el curso al que pertenecen los alumnos. Como se puede apreciar, en el curso de 1er año de odontología se presenta un mayor porcentaje de alumnos que obtuvieron el puntaje que los clasificaba como con Hábitos Saludables y luego se encontraron los alumnos de 4to año. Estas diferencias sin embargo no fueron estadísticamente significativas.

Tabla 1. Características sociodemográficas de la muestra de estudiantes de odontología de la Facultad de Odontología de la Universidad de Chile.

\begin{tabular}{|l|c|c|}
\hline Variables & $\mathbf{n}$ & $\%$ \\
\hline Sexo & & \\
\hline Masculino & 76 & 50.6 \\
\hline Femenino & 74 & 49.4 \\
\hline Año más bajo que cursa & & \\
\hline Primero & 50 & 33.3 \\
\hline Segundo & 38 & 25.3 \\
\hline Tercero & 35 & 23.3 \\
\hline Cuarto & 27 & 18.0 \\
\hline Nivel socioeconómico & & \\
\hline Bajo & 27 & 18.3 \\
\hline Medio & 46 & 31.0 \\
Alto & 75 & 50.6 \\
\hline
\end{tabular}

Tabla 2. Distribución de frecuencia de los hábitos de higiene oral en estudiantes de odontología.

\begin{tabular}{|c|c|c|c|c|}
\hline \multirow[t]{2}{*}{ Variable } & \multicolumn{2}{|c|}{ No saludable } & \multicolumn{2}{|c|}{ Saludable } \\
\hline & $\mathbf{n}$ & $\%$ & $\mathbf{n}$ & $\%$ \\
\hline $\begin{array}{l}\text { Uso de cepillado por lo } \\
\text { menos dos veces al día }\end{array}$ & 3 & 2 & 147 & 98 \\
\hline $\begin{array}{l}\text { Uso de seda dental } \\
\text { por lo menos } 1 \text { vez al día }\end{array}$ & 10 & 62.7 & 56 & 37.3 \\
\hline $\begin{array}{l}\text { Ultima visita fue hace } \\
\text { menos de } 1 \text { año }\end{array}$ & 39 & 26 & 111 & 74 \\
\hline $\begin{array}{l}\text { Índice } \\
\text { Hábitos de Higiene Oral } \\
\text { Saludables }\end{array}$ & 105 & 70 & 45 & 30 \\
\hline
\end{tabular}


Tabla 3. Distribución de frecuencia del índice Hábitos de Higiene Oral Saludables según curso en estudiantes de odontología.

\begin{tabular}{|c|c|c|c|c|}
\hline \multirow{2}{*}{$\begin{array}{l}\text { Índice Hábitos de } \\
\text { Higiene Oral Saludables }\end{array}$} & \multicolumn{4}{|c|}{ Ultimo año que está cursando } \\
\hline & $1^{\text {er }}$ año & $2^{\circ}$ año & $3^{\text {er }}$ año & $4^{\text {to }}$ año \\
\hline & n $\%$ & n $\%$ & n \% & n \% \\
\hline SI & 2040 & 923,7 & 926 & 730 \\
\hline NO & 3060 & 2976,3 & 2674,3 & 2070 \\
\hline Total & 50100 & 38100 & 35100 & 27100 \\
\hline
\end{tabular}

Para evaluar la asociación entre los hábitos de higiene oral saludables y las variables, sexo, año de la carrera que cursa y nivel socioeconómico del grupo familiar se utilizó la prueba de $\mathrm{Chi}^{2}$, y el método de regresión logística simple para la variable edad, no se encontraron asociaciones significativamente estadísticas para ninguna de estas, con un $95 \%$ de confianza (Tabla 4 ).

Tabla 4. Asociación entre hábitos de higiene oral saludables y variables sociodemográficas.

\begin{tabular}{|l|ccc|}
\hline \hline Variable & gl & Chi-cuadrado & P-value \\
\hline Sexo & 148 & 0.182 & 0.669 \\
\hline Año cursado & 148 & 3.622 & 0.305 \\
\hline $\begin{array}{l}\text { Nivel } \\
\text { socioeconómico }\end{array}$ & 148 & 1.42 & 0.491 \\
\hline & OR & IC 95\% & P-value \\
\hline Edad & 1.09 & $0.916-1.318$ & 0.310 \\
\hline
\end{tabular}

\section{DISCUSIÓN}

Los resultados de la presente investigación muestran que los estudiantes de odontología presentan en un alto porcentaje el hábito de cepillado dental, un $98 \%$ de los alumnos se cepilla por lo menos dos veces al día. Este porcentaje es más alto que lo reportado en estudiantes de Odontología en India ${ }^{(8)}$, donde un $54,4 \%$ de los estudiantes de $1 \mathrm{er}$ año y un $50,9 \%$ de los estudiantes de 4 to año relataban que se cepillaban los dientes dos veces al día o más. Por otro lado, Tseveenjav ${ }^{(9)}$, reportó en el año 2002 una frecuencia de cepillado dos veces al día o más en el $81 \%$ de los estudiantes de odontología.

Respecto a visitas al dentista en el ultimo año, observamos que esta fue frecuente, por lo menos una vez en el último año en el $74 \%$ de los estudiantes. Es importante comentar que cuando ingresan los estudiantes de Odontología no se les realiza un examen odontológico, sólo al egreso de ellos en $6^{\text {to }}$ año y este es obligatorio previo a su titulación, grupo que no fue considerado en este estudio. La alta frecuencia de visita al dentista es más alta que lo reportado en la población general de adolescentes chilenos de la provincia de Santiago, $43 \%{ }^{(7)}$. Esta diferencia puede deberse al valor que los estudiantes de Odontología y sus padres le dan a la necesidad del control odontológico profesional. Otro elemento que puede explicar esta diferencia es el nivel socioeconómico más alto que tiene el grupo de estudiantes de Odontología, por ejemplo en ellos un $56 \%$ de sus madres tiene estudios universitarios o técnicos, mientras que en el estudio en adolescentes chilenos sólo un $32 \%$ lo tiene ${ }^{(7)}$.

El uso de seda dental, por lo menos una vez al día, fue reportado por un $37,3 \%$ de los estudiantes, lo que es más alto comparado a los reportado por los estudiantes de India, 4,9\% de 1er año y $12,1 \%$ de 4 to año $^{(8)}$, pero similar a lo reportado por estudiantes de Finlandia, uso de seda dental en un $35 \%$, aunque sólo un $2 \%$ lo hacía diariamente ${ }^{(10)}$.

Con el Índice de Hábitos de Higiene Oral Saludables creado por nosotros, observamos que sólo un $30 \%$ de los estudiantes se clasifica dentro del grupo que se consideraría positivo o con hábitos saludables. Es interesante que al observar la distribución de este índice según año cursado, aunque no se detectaron diferencias estadísticamente significativas, podemos apreciar que los alumnos de 1er año son los que presentan un mayor porcentaje de hábitos saludables, $40 \%$. La razón para esto puede deberse a que durante el 1er año de odontología, los alumnos se encuentran especialmente motivados y se les entrega información sobre prevención de las enfermedades orales, especialmente de caries y enfermedad periodontal, y además específicamente se instruye sobre los buenos hábitos de higiene oral. Esta motivación probablemente puede ir disminuyendo a medida que pasan los años al cambiar el énfasis de los cursos en la adquisición de conocimientos vinculados con el diagnóstico específico, los tratamientos y especialmente en el desarrollo de habilidades clínicas, y con un menor porcentaje de educación en prevención.

Según algunos estudios revisados era posible esperar un mayor frecuencia de hábitos saludables a medida que aumentaban los alumnos en sus cursos de formación odontológica como ha sido reportado en Japón por Kawamura(11), quien lo asocia a la incorporación del curso de odontología preventiva. Sin embargo, es importante considerar que en este estudio comparativo con estudiantes de Finlandia se reportaron diferencias importantes en los hábitos y creencias entre estos dos grupos de estudiantes, por ejemplo en un $56 \%$ de los estudiantes Japoneses creía que los dientes se podían perder aún cepillándose los dientes, comparado con un $2 \%$ que respondió esto en Finlandia. Estas diferencias culturales, que incorporan hábitos, creencias y conocimientos previos, también pueden explicar diferencias en el impacto de la formación odontológica en la adquisición de hábitos saludables por los estudiantes de odontología.

Cuando analizamos los resultados de la variable índice Hábitos de Higiene Oral Saludable por sexo, no observamos diferencias significativas. Esto coincide con lo reportado por estudiantes de Japón y Finlandia

En este estudio tampoco observamos diferencias con la variable nivel socioeconómico. Respecto a esto, puede ser interesante comentar que se ha mencionado una posible dificultad de usar datos autoreportados de indicadores sociales en estudios en adolescentes ${ }^{(7)}$ Sin embargo, estudios previos han demostrado que los adolescentes son capaces de entregar información valida y confiable sobre la posición socioeconómica de su padres ${ }^{(12)}$. Nosotros pensamos que podíamos obtener un porcentaje importante de respuestas omitidas especialmente en la pregunta que consultaba sobre el rango de ingresos del grupo familiar, en parte porque el estudiante podía desconocerlo o por no querer responder a esta pregunta al considerarlo algo privado, sin embargo sólo 2 estudiantes no contestaron esta pregunta.

\section{CONCLUSIONES}

Los estudiantes de odontología presentan buenos hábitos de higiene oral, especialmente en lo que se refiere al cepillado dental y una alta frecuencia de visita al dentista en el último año, desde el inicio de sus estudios en el 1er año de su carrera; además los resultados son mejores que lo reportado en población de adolescentes de Santiago. Sin embargo, el uso de seda dental es bajo, lo que provoca que el índice de hábitos saludables sólo sea de un $30 \%$. No se observa un aumento de adquisición de buenos hábitos a medida que se progresa en los años de estudio de odontología y los alumnos de 1er año son los que presentan en un mayor porcentaje hábitos de higiene oral saludables. Debido a lo anterior y al importante rol social que cumple el odontólogo en los cuidados bucodentales, se hace relevante reforzar la educación y promoción de salud oral durante su proceso de formación profesional.

\section{AGRADECIMIENTOS}

Se agradece a las alumnas de odontología Camila Bravo Castelblanco y Paulina Ledezma Rojas que participaron realizando las encuestas y a Gonzalo Báez Vilches presidente del Centro de Alumnos de la Facultad de Odontología de la Universidad de Chile año 2009 por su colaboración. 


\section{ANEXO 1}

\section{Cuestionario de Hábitos de Higiene Oral.}

$\mathrm{N}^{\circ}$ cuestionario:

Fecha:

A) Hábitos de higiene oral:

Registre en el casillero el número de la opción que mejor represente sus hábitos de higiene oral actuales.

* ¿Cuántas veces al día cepilla sus dientes?

1. Nunca

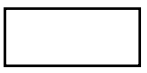

2. Ocasionalmente, no todos los días

3. Una vez al día

4. Dos veces al día

5. Tres veces al día o más

* ¿Cuántas veces al día utiliza seda dental?

1. Nunca

2. Ocasionalmente, no todos los días

3. Una vez al día

4. Dos veces al día

5. Tres veces al día o más

* ¿Hace cuánto tiempo fue su última visita al dentista?

1. No he ido nunca al dentista

2. Hace más de 2 años

3. Entre 1 y 2 años

4. Entre 6 meses y 1 año

5. Hace menos de 6 meses

* ¿Cuántas veces al día utiliza enjuague bucal?

1. Nunca

2. Ocasionalmente, no todos los días

3. Una vez al día

4. Dos veces al día

5. Tres veces al día o más

B) Características sociodemográficas:

* Escolaridad de la madre

1. Sin educación

2. Básica completa o incompleta

3. Media completa o incompleta

4. Técnica completa o incompleta

5. Universitaria completa o incompleta

* Escolaridad del padre

1. Sin educación

2. Básica completa o incompleta

3. Media completa o incompleta

4. Técnica completa o incompleta

5. Universitaria completa o incompleta 
* Ingreso total mensual del grupo familiar

1. Menos de $\$ 160.000$

2. Entre $\$ 161.000$ y $\$ 500.000$

3. Entre $\$ 501.000$ y $\$ 1.000 .000$

4. Entre $\$ 1.000 .001$ y $\$ 2.000 .000$

5. Más de $\$ 2.000 .000$

C) Datos personales:

* Año de ingreso a la carrera

* Indique el año más bajo que esté cursando

1. Primer año

2. Segundo año

3. Tercer año

4. Cuarto año

5. Quinto año

* Sexo

1. Masculino

2. Femenino

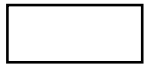

* $\quad$ Edad

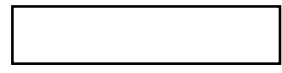

años 


\section{ANEXO 2}

Distribución de los resultados de Índice de Hábitos de Higiene Oral de acuerdo a características sociodemográficas y socioeconómicas en la muestra estudiada.

*Índice Hábitos de Higiene Oral

Saludables $\%$

No saludables $\%$

Escolaridad de la Madre

Sin educación $(0 \%)$

Básica completa o incompleta $(2 \%)$

Media completa o incompleta $(20.7 \%)$

Técnica completa o incompleta $(26 \%)$

Universitaria completa o incompleta $(51.3 \%)$

Escolaridad del Padre

Sin educación $(0 \%)$

Básica completa o incompleta $(2 \%)$

Media completa o incompleta (21.3\%)

Tecnica completa o incompleta (14.7\%)

Universitaria completa o incompleta (62\%)

Ingreso familiar mensual (miles \$)

Menos de $160(3.3 \%)$

Entre 160 y $500(20 \%)$

Entre 501 y 1000 (19.3\%)

Entre 1 millón y2 millones (28.7\%)

Mas de 2 millón (27.3\%)

Año ingreso a la carrera

$2004(2.7 \%)$

$2005(2.7 \%)$

$2006(20 \%)$

$2007(18.7 \%)$

$2008(24.7 \%)$

$2009(31.3 \%)$

Año carrera cursando

Primer $(33.3 \%)$

Segundo $(25.3 \%)$

Tercero $(23.3 \%)$

Cuarto $(18 \%)$

Sexo

Masculino (50.7\%)

Femenino (49.3\%)

Edad

$<20$ años $(41.3 \%)$

$>=20$ años $(58.7 \%)$
2.2

22.2

35.6

40

2.2

20

17.8

60

24.4

22.2

33.3

17.8

-

6.7

11.1

17.8

22.2

42.2

44.4

20

20

15.6

53.3

46.7

55.6

44.4
1.9

20

21.9

56.2

1.9

21.9

13.3

62.9

4.8

18.1

18.1

26.7

31.4

3.8

23.8

19

25.7

26.7

28.6

27.6

24.8

19.1

49.5

50.5

40

60

*Índice hábitos de higiene oral saludables, este es un índice generado a partir de la variable frecuencia cepillado (por lo menos 2 veces al día), variable seda dental (por lo menos 1 vez al dia) y visita al dentista (por lo menos 1 vez al año), los individuos que cumplían con las tres categorías fueron clasificados como con hábitos de higiene oral saludables. 


\section{ANEXO 3}

Distribución de los resultados de los componentes de Hábitos de Higiene Oral.

¿Cuántas veces al día cepilla sus dientes?

1. Nunca

2. Ocasionalmente, no todos los días

3. Una vez al día

4. Dos veces al día

5. Tres veces al día o más

¿Cuántas veces al día utiliza seda dental?

1. Nunca

2. Ocasionalmente, no todos los días

3. Una vez al día

4. Dos veces al día

5. Tres veces al día o más

¿Hace cuánto tiempo fue su última visita al dentista?

1. No he ido nunca al dentista

2. Hace más de 2 años

3. Entre 1 y 2 años

4. Entre 6 meses y 1 año

5. Hace menos de 6 meses

¿Cuántas veces al día utiliza enjuague bucal?

1. Nunca

2. Ocasionalmente, no todos los días

3. Una vez al día

4. Dos veces al día

5. Tres veces al día o más n

$\%$

0.67

1.33

26

72

12.7

50

33.3

4

6

21

39

72

14

26

48

22.7

30.7

27.3

16

2.7 


\section{REFERENCIAS BIBLIOGRÁFICAS}

1. Ministerio de Salud. Encuesta Nacional de Salud. Ministerio de Salud. 2003. Búsqueda 8 de Abril 2009

2. Gamonal JA, Lopez NJ, Aranda W. Periodontal conditions and treatment needs, by CPITN, in the 35-44 and 65-74 year-old population in Santiago, Chile, Int Dent J 1998. 48(2): 96-103.

3. Petersen PE, Bourgeois D, Ogawa H, Estupinan-Day S, Ndiaye C. The global burden of oral diseases and risks to oral health. Bulletin of the World Health Organization (WHO) Global Oral Health Programme, Department for Chronic Disease and Health Promotion.2006.

4. Rivas J. Hábitos y conocimientos de higiene bucodental de los pacientes que acuden por atención odontológica a la clínica periférica no. 1, del IHSS. Primer trimestre 2006. Tesis para optar al grado de Magíster en Salud Publica.2006 Nicaragua.

5. Abraham NJ, Cirincione UK, Glass RT. Dentist's and dental higyenists's attitude toward tooth brush replacement and maintenance. Clin Prev Dent.1990. 12: 28-33.

6. Uitenbroek DG, Schaub RMH, Troomp JAH, Kant JH. Dental hygienist influence on patients's knowledge, motivation, self care, and perception of change. Community Dent Oral Epidemiol. 1989. 17: 87-90.
7. Lopez $R$ and Baelum V. Factors associated with dental attendance among adolescent in Santiago, Chile. BMC Oral Health.2007. 7:4.

8. Sharda AJ, Shetty S. A comparative study of oral health knowledge, attitude and behaviour of first and final year dental students of Udaipur city, Rajasthan, India. 2008. 6(4);347-53.

9. Tseveenjav B, Vehkalahti M, Murtomaa H. Preventive practice of Mongolian dental students Eur J Dent Educ. 2002:;6(2):74-8.

10. Murtomaa H, Turtola L, Rytömaa I. Use of dental floss by Finnish students. J Clin Periodontol.1984. 11(7):443-7.

11. Kawamura M, Honkala E, Widström E, Komabayashi T. Cross-cultural differences of self-reported oral health behaviour in Japanese and Finnish dental students. Int Dent J. 2000; 50(1):46-50.

12. Lien N, Friestad C, Klepp KI J. Adolescents' proxy reports of parents' socioeconomic status: How valid are they? Epidemiol Community Health. 2001. 55(10):731-7. 